\title{
Generalized Abel Integral Operators on Spaces with Rooney Weights
}

\author{
F. PENZEL and I. SPITKOVSKY
}

We prove a Fredholm criterion for generalized Abel integral equations on the half-line on weighted $L_{p}$-spaces for a class of weight functions introduced by Rooney. We obtain the equivalence of these integral equations to singular integral equations.

Key words: Abel integral equations, singular integral equations, general weight functions AMS classification: $45 \mathrm{E} 10,45 \mathrm{~F} 10$

\section{Introduction}

In this paper we study the Fredholm properties of the system of generalized Abel integral equations

$$
\frac{a(x) x^{-\alpha}}{\Gamma(\alpha)} \int_{0}^{x} \frac{u(t)}{(x-t)^{1-\alpha}} d t+\frac{b(x) x^{-\alpha}}{\Gamma(\alpha)} \int_{x}^{\infty} \frac{u(t)}{(t-x)^{1-\alpha}} d t=g(x)
$$

in weighted $L^{p}$-spaces. For a survey of the history, applications, and the theory of this type of equations we refer the reader to [8: p. 441], [3: pp. $61-121],[5]$ and the literature cited therein. In [5] the theory is developed for weight functions of the type $\rho_{\mu}(x)=x^{\mu}$. Recent results in $[1,9]$ for singular integral equations with general weights are used bere to study the Fredholm criteria of the integral operator defined by (1) and its equivalence to a singular integral equation. In Section 3 on generalizations we point out some interesting results for generalized Abel integral equations on a compact interval and for the case of systems.

Acknowledgement: The main part of this paper has been prepared during the visit of the second author to the University of Darmstadt in the Summer of 1992 . We would like to thank Prof. Meister who made this visit possible.

\section{Preliminaries}

The following notation due to [7] is used.

Definition 1.1: Let $w$ be a non-negative locally integrable function on $(0, \infty)$. If $\mu \in \mathbb{R}$ and $1 \leq p<\infty$, we define $\mathcal{L}_{w, \mu, p}$ to consist of all those complex-valued functions,

F. Penzel: Tochn. Hochschule, FB Math., SchloBgartzenstr. 7, D - 64289 Darmstadt

I. Spitkoveky: Collego of William and Mary, Dop. Math., Williamsburg. Virginia 2318S. USA 
which are measurable on $(0, \infty)$ and which satisfy

$$
\|f\|_{w, \mu, p}=\left\{\int_{0}^{\infty} w(x)\left|x^{\mu} f(x)\right|^{p} \frac{d x}{x}\right\}^{1 / p}<\infty .
$$

The definition of a Rooney weight [7] is given in the following

Definition 1.2: Suppose $w$ is a non-negative locally integrable function on $(0, \infty)$ and $1<p<\infty$. We say $w$ is a Rooney weight if there is a constant $K$ such that, for all positive real $a, b$ with $a<b$,

$$
\left\{\int_{a}^{b} w(x) \frac{d x}{x}\right\}\left\{\int_{a}^{b} w^{1 /(p-1)}(x) \frac{d x}{x}\right\}^{p-1} \leq K\left(\log \frac{b}{a}\right)^{p} .
$$

If $w$ satisfies this inequality, we write $w \in \mathcal{A}_{p}$.

Definition 1.3: For given $\alpha>0$ let $I_{\alpha}$ and $J_{\alpha}$ be the fractional integral operators

$$
\left(I_{\alpha} f\right)(x)=\frac{x^{-\alpha}}{\Gamma(\alpha)} \int_{0}^{x} \frac{f(t)}{(x-t)^{1-\alpha}} d t \quad \text { and } \quad\left(J_{\alpha} f\right)(x)=\frac{x^{-\alpha}}{\Gamma(\alpha)} \int_{x}^{\infty} \frac{f(t)}{(t-x)^{1-\alpha}} d t
$$

for $x>0$.

In [7: pp. $260-261]$ the following result was obtained.

Theorem 1.4: Suppose $1<p<\infty, 0<\alpha<\mu<1$, and $w \in \mathcal{A}_{p}$. Then $I_{\alpha}$ and $J_{\alpha}$ are compact, injective operators mapping $\mathcal{L}_{w, \mu, p}$ into itself. It holds $I_{\alpha}\left(\dot{\mathcal{L}}_{w, \mu, p}\right)=J_{\alpha}\left(\mathcal{L}_{w, \mu, p}\right)$. The operators $I_{\alpha}^{-1} J_{\alpha}$ and $J_{\alpha}^{-1} I_{\alpha}$ map $\mathcal{L}_{w, \mu, p}$ one-to-one and onto itself.

Definition 1.5: A function $a:[0, \infty) \rightarrow \mathbb{C}$ is called Hölder-continuous of order $\beta, 0<\beta \leq 1$, if there exists a constant $C>0$ such that

$$
|a(x)-a(y)| \leq C \frac{|x-y|^{\beta}}{(1+x)^{\beta}(1+y)^{\beta}}
$$

for all $x, y>0$. The set of all Hölder-continuous functions of order $\beta$ is denoted by $H^{\beta}\left(\overline{\mathbb{R}}^{+}\right)$.

Definition 1.6: The singular integral operator $S_{\gamma}$ on the half-line with weight $x^{\gamma}$ is defined by

$$
\left(S_{\gamma} f\right)(x)=\frac{1}{\pi i} \int_{0}^{\infty}\left(\frac{y}{x}\right)^{\gamma} \frac{f(y)}{y-x} d y .
$$

The integral here has to be understood in the sense of a Cauchy principal value. 


\section{Fredholm properties of generalized Abel integral equations}

The following two lemmata were obtained in [5: Proposition 4.3 and p. 614] for the special case $w \equiv 1$.

Lemma 2.1: Suppose $0<\alpha<\mu<1, \alpha<\beta, 1<p<\infty, a \in H^{\beta}\left(\overline{I R}^{+}\right)$and $w \in \mathcal{A}_{p}$. Then the operator $I_{\alpha}^{-1}\left(a I_{\alpha}-I_{\alpha} a\right)$ defined for functions in $C_{0}^{\infty}\left(\mathbb{R}^{+}\right)$can be continuously extended to a compact operator on $\mathcal{L}_{w, \mu, p}$.

Proof: By use of estimate (2) the following one for functions $a \in H^{\beta}\left(\bar{R}^{+}\right)$and $g \in \mathcal{L}_{1, \mu, p}$ was obtained in [4: pp. $20-21$ ]:

$$
\left|I_{\alpha}^{-1}\left(a I_{\alpha} g-I_{\alpha} a g\right)\right|(x) \leq C\left(\frac{x}{(1+x)^{2}}\right)^{\beta / 2} x^{-\beta / 2} \int_{0}^{x}(x-u)^{\beta-\alpha-1} u^{\alpha-\beta / 2}\left|I_{\alpha} g\right|(u) d u .
$$

This estimate was proved by use of an explicit representation of the operator $I_{\alpha}^{-1}$ and an estimate of the kernel of the integral operator $I_{\alpha}^{-1}\left(a I_{\alpha}-I_{\alpha} a\right)$. Because of Theorem 1.4 the continuity of $I_{\alpha}$ follows, and it is sufficient to prove that the operator

$$
K h(x)=\left(\frac{x}{(1+x)^{2}}\right)^{\beta / 2} \int_{0}^{x}\left(\frac{x}{u}-1\right)^{\beta-\alpha-1}\left(\frac{x}{u}\right)^{-\beta / 2}|h(u)| \frac{d u}{u}
$$

has a compact extension to $\mathcal{L}_{w, \mu, p}$. If we have verified that $K$ is compact; then the assertion of the lemma follows from the fact that an integral operator $L: \mathcal{L}_{w, \mu, p} \rightarrow \mathcal{L}_{w, \mu, p}$ defined by $L g(x)=\int_{0}^{\infty} k(x, t) g(t) d t$ is compact, if a compact operator $M: \mathcal{L}_{w, \mu, p} \rightarrow \mathcal{L}_{w, \mu, p}$ exists such that $|L g(x)| \leq(M(|g|))(x)$ for all $x>0$ and all $g \in \mathcal{L}_{w, \mu, p}$ [10: p. 94]. Furthermore, the operator $K: \mathcal{L}_{w, \mu, p} \rightarrow \mathcal{L}_{w, \mu, p}$ is the composition of a Mellin convolution operator with an operator of multiplication by a Hölder-continuous function which vanishes at $x=0$ and which vanishes if $x \rightarrow \infty$. Therefore the operator $K$ may be approximated in the operator norm by operators

$$
K_{\Phi, \Psi} h(x)=\Psi(x) \int_{0}^{x} \Phi\left(\frac{x}{u}\right)|h(u)| \frac{d u}{u}
$$

with $\Phi, \Psi \in C_{0}^{\infty}\left(\mathbb{R}^{+}\right)$. The set of compact operators in $\mathcal{L}_{w, \mu, p}$ is closed, therefore it is sufficient to prove the compactness of all operators $K_{\Phi, \Psi}$. The compactness of $K_{\Phi, \Psi}$ is equivalent to that of the operator $L_{\Phi, \Psi}: L_{p}\left(\mathbb{R}^{+}\right) \rightarrow L_{p}\left(\mathbb{R}^{+}\right)$defined by

$$
L_{\$, \Psi} h(x)=\Psi(x) \int_{0}^{x} \Phi\left(\frac{x}{u}\right)\left\{\frac{w(x)}{w(u)}\right\}^{1 / p}\left(\frac{x}{u}\right)^{\mu-1 / p}|h(u)| \frac{d u}{u} .
$$

Moreover, by an application of the criterion of Hille-Tamarkin for operators in $L_{p}$ it is sufficient to prove that the following integral exists:

$$
I(\Phi, \Psi)=\int_{0}^{\infty}\left\{\int_{0}^{x}\left|\Psi(x)\left\{\frac{w(x)}{w(u)}\right\}^{1 / p} \Phi\left(\frac{x}{u}\right)\left(\frac{x}{u}\right)^{\mu-1 / p} \frac{1}{u}\right|^{p^{\prime}} d u\right\}^{p / p^{\prime}} d x .
$$


If $n \in \mathbb{N}$ is such that the supports of $\Phi$ and $\Psi$ are subsets of $\left(\frac{1}{n}, n\right)$, then it is easily proved that there are numbers $C_{1}, C_{2}>0$ which depend only on $\Phi$ and $\Psi$ such that

$$
\begin{aligned}
I(\Phi, \Psi) & \leq C_{1} \int_{1 / n}^{n} \int_{1 / n^{2}}^{x} \mid\left\{\left.\left\{\frac{w(x)}{w(u)}\right\}^{1 / p}\left(\frac{x}{u}\right)^{\mu-1 / p} \frac{1}{u}\right|^{p^{\prime}} d u\right\}^{p^{\prime}-1} d x \\
& \leq C_{2} \int_{1 / n}^{n} w(x) \frac{d x}{x}\left\{\int_{1 / n^{2}}^{n} w^{1 /(p-1)}(u) \frac{d u}{u}\right\}^{p-1}<\infty .
\end{aligned}
$$

The latter follows from Definition 1.2

Lemma 2.2: Suppose $0<\alpha<\mu<1,1<p<\infty, w \in \mathcal{A}_{p}$, and $f \in \dot{L}_{w, \mu, p}$. Then

$$
I_{\alpha} f=-\cos (\pi(1-\alpha)) J_{\alpha} f-i \sin (\pi(1-\alpha)) S_{0} J_{\alpha} f
$$

Proof: In the case $w \equiv 1$ relation (3) is easily computed from the formulas (8.1) and (8.2) in [5]. From [7: p. 258] we obtain that $C_{0}^{\infty}\left(\mathbb{R}^{+}\right)$is a dense subset of $\dot{L}_{w, \mu, p}$ if $w \in \mathcal{A}_{p}$, furthermore the results in [7] imply the continuity of the operators in both sides of relation (3). By continuous extension we can conclude our result

Remark: From Lemma 2.2 it is obvious that the equation (1) can be reduced by substitution (3) to the singular integral equation

$$
\left(c I+d S_{0}\right) v=g
$$

where $c, d$, and $v$ are given by

$$
c=b-\cos (\pi(1-\alpha)) a, \quad d=-i \sin (\pi(1-\alpha)) a, \quad v=J_{\alpha} u .
$$

The spectrum and essential spectrum (which happen in this case to be the same) of the operator $S_{0}$ in spaces $\dot{L}_{w, \mu, p}$ were described in [2].

Theorem 2.3: Suppose $0<\mu<1,0<\beta<1,1<p<\infty, w \in \mathcal{A}_{p}$, and $c, d \in$ $H^{\beta}\left(\overline{\mathbb{R}}^{+}\right)$. Then the operator $A_{0}=c I+d S_{0}: \dot{\mathcal{L}}_{w, \mu, p} \rightarrow \dot{L}_{w, \mu, p}$ is of Fredholm type if and only if

$$
c(x) \pm d(x) \neq 0 \quad\left(x \in \bar{R}^{+}\right) .
$$

as well as

$$
\arg \frac{c(0)-d(0)}{c(0)+d(0)} \neq 2 \pi \mu \quad \text { and } \quad \arg \frac{c(\infty)-d(\infty)}{c(\infty)+d(\infty)} \neq 2 \pi \mu .
$$

Proof: Consider the space $\tilde{\mathcal{L}}_{w, \mu, p}$ of complex-valued functions defined on $\mathbb{R}$ and such that $\|f\|:=\left(\int_{-\infty}^{\infty} w(|x|)\left|x^{\mu} f(x)\right|^{p} \frac{d x}{|x|}\right)^{1 / p}<\infty$. Of course, $\overline{\mathcal{L}}_{w, \mu, p}$ is a direct sum of $\mathcal{L}_{w, \mu, p}$ with its image $\mathcal{L}_{w, \mu, p}^{\prime}$ under the reflection $(T f)(x)=f(-x)$. The singular integral operator $S$ defined by

$$
(S f)(x)=\frac{1}{\pi i} \int_{-\infty}^{\infty} \frac{f(y)}{y-x} d y
$$


is bounded in $\tilde{\mathcal{L}}_{w, \mu, p}[7:$ p. 261]. Put

$$
\tilde{c}(x)=\left\{\begin{array}{ll}
c(x) & \text { for } x>0 \\
1 & \text { for } x<0
\end{array} \quad \text { and } \quad \tilde{d}(x)=\left\{\begin{array}{ll}
d(x) & \text { for } x>0 \\
0 & \text { for } x<0
\end{array} .\right.\right.
$$

Then $A=\dot{c} I+\dot{d} S$ is the direct sum of $A_{0}$ with the identity operator on $\mathcal{L}_{w, \mu, p}^{\prime}$. Hence, $A$ and $A_{0}$ have the same Fredholm properties. But $A$ is a singular integral operator on the space $\overline{\mathcal{L}}_{w, \mu, p}=L_{p, \rho}(\mathbb{R})$ with the Muckenhoupt weight $\rho(x)=w(|x|)|x|^{\mu-\frac{1}{p}}$ and has coefficients which are continuous on $[-\infty, 0]$ and $[0, \infty]$. The desired criterion now follows from [1: Theorem 2.10] and [2: Lemma 3.3]

As a consequence we obtain the following result for the equation (1).

Teorem 2.4: Suppose $0<\alpha<\beta<1,0<\alpha<\mu<1,1<p<\infty, w \in \mathcal{A}_{p}$, and $a, b \in H^{\beta}\left(\overline{\mathbb{R}}^{+}\right)$. Then the following two propositions hold.

(i) The operator $I_{\alpha}^{-1}\left(a I_{\alpha}+b J_{\alpha}\right): \dot{\mathcal{L}}_{w, \mu, p} \rightarrow \dot{\mathcal{L}}_{w, \mu, p}$ is of Fredholm type if and only if the functions $c$ and $d$ defined by (5) fulfill the conditions (6) and (7).

(ii) If $g \in \mathcal{L}_{w, \mu, p}$ is in the range of the operator $I_{\alpha}: \dot{\mathcal{L}}_{w, \mu, p} \rightarrow \dot{\mathcal{L}}_{w, \mu, p}$ and equation (4) has a solution $v \in \mathcal{L}_{w, \mu, p}$, then $u=J_{\alpha}^{-1} v$ is a solution of equation (1).

Proof: As a consequence of Lemma 2.1 the operator $I_{\alpha}^{-1}\left(a I_{\alpha}+b J_{\alpha}\right)-\left(a I+b I_{\alpha}^{-1} J_{\alpha}\right)$ is compact. By Lemma 2.2 we obtain $a I+b I_{\alpha}^{-1} J_{\alpha}=c I+d S_{0}$, therefore proposition (i) follows from Theorem $2.3 /(\mathrm{i})$.

In the case $w \equiv 1$ it was proved in [5: pp. 612 - 613] that there exists a regularizer of the operator $c I+d S_{0}$ of the form

$$
R=\sum_{l=1}^{4} a_{l} S_{\gamma_{l}} b_{l}+\sum_{m=1}^{2} c_{m} \omega S_{0} \omega^{-1} d_{m},
$$

with numbers $\gamma_{l} \in(-\mu, 1-\mu)$ (in our notation here), functions $a_{l}, b_{l} \in H^{\beta}\left(\overline{I R}^{+}\right), \omega(t)=$ $t^{\gamma_{1}}(1+t)^{\gamma_{1}-\gamma_{3}}$ and functions $c_{m}, d_{m}$ satisfying supp $c_{m} \cap$ supp $d_{m}=0$. This operator is bounded on $\mathcal{L}_{w, \mu, p}$, and even on $\mathcal{L}_{w^{1+\cdot, \mu, p}}$ for $\epsilon$ sufficiently small. Hence, the operators $R A_{0}-I$ and $A_{0} R-I$ are compact on $\dot{\mathcal{L}}_{1, \mu, p}$ and bounded on $\mathcal{L}_{w^{1+\rho, \mu, p}}$. It then follows from the interpolation theorem for compact operators [6] that $R A_{0}-I$ and $A_{0} R-I$ are compact on $\mathcal{L}_{w, \mu, p}$. In other words, $R$ is a regularizer of $A_{0}$ on $\mathcal{L}_{w, \mu, p}$. Therefore it is sufficient to prove that this regularizer leaves the space $I_{\alpha}\left(\mathcal{L}_{w, \mu, p}\right)$ invariant. By Lemma 2.1 multiplication with a function from $H^{\beta}\left(\overline{I R}^{+}\right)$maps the set $I_{\alpha}\left(\mathcal{L}_{w, \mu, p}\right)$ into itself. Because the operators $S_{-\gamma_{t}}$ are Mellin convolutions it follows from the results in [7] that these operators leave the space $I_{\alpha}\left(\mathcal{L}_{w, \mu, p}\right)$ invariant. Thus the first sum maps $I_{\alpha}\left(\mathcal{L}_{w, \mu, p}\right)$ into itself.

The second sum represents a "smoothing" operator. It was proved in the case $w \equiv 1$ in [5] that those operators map $\mathcal{L}_{1, \mu, p}$ continuously into $I_{\alpha}\left(\mathcal{L}_{1, \mu, p}\right)$. It is easily seen that these arguments carry over to the case of arbitrary $w \in \mathcal{A}_{p}$. All (possible) solutions of equation (4) are obtained by application of the regularizer of $c I+d S_{0}$ and, in addition, if $g$ in equation (4) is in the range of $I_{\alpha}\left(\mathcal{L}_{w, \mu, p}\right)=J_{\alpha}\left(\mathcal{L}_{w, \mu, p}\right)$, then we proved that the same holds for the solution $v$ 


\section{Generalizations}

We would like to mention two generalizations.

First, our results may be extended to systems of generalized Abel integral equations in the case where we impose the stronger assumption that the coefficients in (1) be Höldercontinuously differentiable. A combination of the results obtained here and those of $[9$ : Theorem 3.1] and of [5: Theorem 8.1] is then obvious.

Secondly, we obtain new results for the Fredholm property and the solution of generalize Abel integral equations on a compact interval $[r, R]$. If we use the substitution $t=\frac{\tau-r}{R-r}$ and $x=\frac{y-r}{R-y}$, then the equation

$$
\frac{a(x) x^{-\alpha}}{\Gamma(\alpha)} \int_{0}^{x} \frac{u(t)}{(x-t)^{1-\alpha}} d t+\frac{b(x) x^{-\alpha}}{\Gamma(\alpha)} \int_{x}^{\infty} \frac{u(t)}{(t-x)^{1-\alpha}} d t=\frac{x^{-\alpha}}{\Gamma(\alpha)} \int_{0}^{x} \frac{: h(t)}{(x-t)^{1-\alpha}} d t
$$

is transformed into

$$
\begin{gathered}
\frac{A(y)}{\Gamma(\alpha)} \int_{\tau}^{y} \frac{u^{*}(\tau)}{(R-\tau)^{1+\alpha}(y-\tau)^{1-\alpha}} d \tau+\frac{B(y)}{\Gamma(\alpha)} \int_{y}^{\infty} \frac{u^{*}(\tau)}{(R-\tau)^{1+\alpha}(\tau-y)^{1-\alpha}} d \tau \\
=\frac{1}{\Gamma(\alpha)} \int_{\tau}^{y} \frac{h^{*}(\tau)}{(R-\tau)^{1+\alpha}(y-\tau)^{1-\alpha}} d \tau
\end{gathered}
$$

with

$$
A(y)=a\left(\frac{y-r}{R-y}\right) \quad \text { and } \quad B(y)=b\left(\frac{y-r}{R-y}\right)
$$

as well as

$$
u^{*}(\tau)=u\left(\frac{\tau-r}{R-\tau}\right) \quad \text { and } \quad h^{*}(\tau)=h\left(\frac{\tau-r}{R-\tau}\right)
$$

Furthermore we have that

$$
\int_{0}^{\infty} w(x)\left|x^{\mu} u(x)\right|^{p} \frac{d x}{x}=\int_{r}^{R} w\left(\frac{y-r}{R-y}\right)\left|u^{*}(y)\right|^{p} \frac{(y-r)^{\mu p}}{(R-y)^{3+\mu p}} d y
$$

and that $A$ and $B$ are Hölder continuous of order $\beta$ on the closed interval $[r, R]$ if and only if $a, b \in H^{\beta}\left(\overline{I R}^{+}\right)$. From these calculations the results of Theorem 2.4 can be easily used for a discussion of equation (9). Even in non-weighted $L^{P}$-spaces $L^{P}([r, R])$ this discussion was not possible from the theory of equation $(1)$ in the space $\dot{\mathcal{L}}_{1, \mu, p}$.

\section{References}

[1] BoetTChER, A. and I. SPITKovSKY: Wiener-Hopf integral operators with PC symbols on spaces with Muckenhoupt weight. Revista Mat. Iberoamer. (to appear).

[2] BoetTCher, A. and I. SpITkovSKY: On a theorem of Rooney concerning the spectrum of a singular integnal operator. Z. Anal. Anw. 12 (1993), 93 - 94.

[3] Gorenplo, R. and S. Vessella: Abel Integral Equations. Lect. Notes Math. 1461 (1991), 1 - 215. 
[4] Penzel, F.: Zur Theorie verallgemeinerter Abelscher Integralgleichungen. PhD-thesis, D17. Darmstadt: Technische Hochschule 1986.

[5] Penzel, F.: On the theory of generalized Abel integral equations on the half-line. Int. Equ. Oper. Theory 10 (1987), $595-620$.

[6] SHARPLEY, R.: Interpolation theorems for compact operators. Ind. Univ. Math. J. 22 (1973), $965-984$.

[7] Rooney, P. G.: Multipliers for the Mellin transformation. Can. Math. Bull. 25 (1982), $257 \cdot 262$.

[8] Samko, S. G., KIlbas, A. A. and O. I. Marichev: Integrals and Derivatives of Fractional Order and Some of Their Applications (in Russian). Minsk: Nauka i Technika 1987.

[9] SPIтkovsKy, I.: Singular integral operators with PC symbols on the spaces with general weights. J. Funct. Anal. 105 (1992), $129-143$.

[10] Zabreyko, P. P.: Integral Equations. Groningen: Nordhoff 1975.

Received: 25.11 .1992 